\title{
Ultrafast dynamics of water in cationic micelles
}

\author{
Adriaan M. Dokter, Sander Woutersen, and Huib J. Bakker \\ FOM Institute for Atomic and Molecular Physics, Kruislaan 407, 1098 SJ Amsterdam, The Netherlands
}

(Received 22 December 2006; accepted 29 January 2007; published online 30 March 2007)

\begin{abstract}
The effect of confinement on the dynamical properties of liquid water is investigated for water enclosed in cationic reverse micelles. The authors performed mid-infrared ultrafast pump-probe spectroscopy on the $\mathrm{OH}$-stretch vibration of isotopically diluted $\mathrm{HDO}$ in $\mathrm{D}_{2} \mathrm{O}$ in cetyltrimethylammonium bromide $(\mathrm{CTAB})$ reverse micelles of various sizes. The authors observe that the surfactant counterions are inhomogeneously distributed throughout the reverse micelle, and that regions of extreme salinity occur near the interfacial Stern layer. The authors find that the water molecules in the core of the micelles show similar orientational dynamics as bulk water, and that water molecules in the counterion-rich interfacial region are much less mobile. An explicit comparison is made with the dynamics of water confined in anionic sodium bis(2-ethythexyl) sulfosuccinate (AOT) reverse micelles. The authors find that interfacial water in cationic CTAB reverse micelles has a higher orientational mobility than water in anionic AOT reverse micelles. (C) 2007 American Institute of Physics. [DOI: 10.1063/1.2721527]
\end{abstract}

\section{INTRODUCTION}

Water plays a crucial role as a solvent in many systems found throughout biology and chemistry. Often such systems do not contain water as a bulk liquid, but as a limited number of water molecules in a confined environment. In bulk water, $\mathrm{H}_{2} \mathrm{O}$ molecules arrange in a dynamical hydrogen bond network, in which most molecules are tetrahedrally coordinated to four other water molecules. Nanoconfinement can have a strong effect on the strength, the structure, and the mobility of the network.

Reverse micelles have proven to be excellent model systems for studying the properties of water in strong confinement. ${ }^{1}$ Such micelles consist of small aqueous droplets that are coated by a layer of surfactant molecules and are dispersed in an apolar solvent. The hydrophilic head groups of the surfactant molecules point toward the micelle interior, while their hydrophobic tails point outward to the exterior solvent. For a large variety of surfactants and solvents, the size of the micelles increases monotonically with the water content (conventionally denoted by the parameter $w_{0}$, defined as the molar ratio [water $] /[$ surfactant]). This property makes it very easy to vary the degree of confinement of water inside the micelles.

The infrared absorption spectrum of water inside reverse micelles differs significantly from that of bulk water, as was shown by Fourier-transform infrared absorption studies. ${ }^{2-6}$ The infrared line shapes deviate most strongly from bulk water for very small micelles and converge to the bulk spectra for large micelles. Based on these observations, the interior water in reverse micelles is thought to consist of different types of molecules. Water molecules in the outer shell of the micelle experience different surroundings and hydrogen bonding than water molecules in the core of the droplets, and have therefore different spectral signatures.

Different types of surfactants can be used to prepare reverse micelles, forming different environments for enclosed water in the micelle core. Most surfactants are classified as either anionic, cationic, or neutral, referring to the nature of their hydrophilic head group. ${ }^{7}$ Ionic surfactants have counterions of opposite charge associating with their charged head groups. For normal micelles dispersed in water it was found that most of these counterions reside at the surfactant-water interface ${ }^{8,9}$ and do not enter the bulk solution. At the interface a so-called Stern layer forms, ${ }^{9}$ densely packed with counterions, surfactant head groups, and limited amounts of water. Analogously, a similar interfacial region is expected to form in large reverse micelles.

The dynamics of confined water in reverse micelles was first investigated by measuring the ${ }^{1} \mathrm{H}$ and ${ }^{17} \mathrm{O}$ spin relaxation of water using nuclear magnetic resonance (NMR). ${ }^{6,10-15}$ This work clearly showed that the mobility of water slows down as a result of confinement. Because different types of water will rapidly exchange roles on the time scale of NMR $\left(10^{-4} \mathrm{~s}\right)$, only information on the average mobility of water within the micelles could be obtained. The same holds for more recent quasielastic neutron scattering studies. ${ }^{16,17}$ From these studies it is therefore not clear whether all of the water molecules in the micelles have a lower mobility or only those molecules at the micellar interface.

The development of ultrafast optical techniques has allowed studies on water dynamics in which the time scales of molecular motions and vibrational relaxation can be resolved. Several ultrafast experiments were recently reported in literature, aimed at a better understanding of the ultrafast dynamics of strongly confined water. ${ }^{18-27}$ Almost all studies have focused on micellar systems containing the anionic surfactant sodium bis(2-ethylhexyl) sulfosuccinate (AOT). This system has been well characterized and is known to form monodisperse reverse micelles in a wide range of sizes. Early ultrafast studies used small probe molecules to examine the interior water pool of reverse micelles. ${ }^{22}$ More recently, mid- 
infrared pump-probe spectroscopy was used to study the dynamics of the $\mathrm{OH}$-stretch vibration of water directly. ${ }^{23-27}$ For anionic AOT micelles, it is found that water molecules in the outer shell of the micelles are strongly immobilized by the confining interface, while molecules in the micelle core behave much like bulk water. ${ }^{27}$ The mobility of water molecules is therefore strongly inhomogeneous within the droplets, as confirmed by detailed molecular dynamics (MD) simulations. $^{17,28}$

Because specific interface-water interactions are expected to be very important in explaining the different behavior of interfacial water compared to bulk water, it is interesting to see how other surfactants than AOT will affect water dynamics within reverse micelles. The present work aims at studying ultrafast water dynamics confined in cationic micelles using the surfactant salt cetyltrimethylammonium bromide (CTAB). We will explicitly compare the new results to earlier results obtained for anionic reverse micelle systems, in particular, AOT reverse micelles.

\section{MATERIALS AND METHODS}

CTAB is one of the most widely used surfactant salts for making cationic reverse micelles. The microstructure of CTAB reverse micelles has been relatively well investigated by time-resolved fluorescence quenching (TRFQ), dynamic light scattering, and conductivity and self-diffusion measurements. ${ }^{6,129}$ In all studies, the water content of the micelles was shown to increase monotonically with the parameter $w_{0}=\left[\mathrm{H}_{2} \mathrm{O}\right] /[\mathrm{CTAB}]$.

We used both surfactant salts CTAB and cetyltrimethylammonium chloride (CTAC) in preparing cationic micelles. For CTAB, a hexadecyltrimethylammonium cation $\left(\mathrm{CTA}^{+}\right)$ acts as the surfactant molecule, of which the hexadecyl group points toward the apolar solvent and the ammonium group toward the water pool. Bromide anions $\left(\mathrm{Br}^{-}\right)$are present as the surfactant counterions. The surfactant salt CTAC consists of the same $\mathrm{CTA}^{+}$cation; however, the bromide ions have been exchanged for chloride ions. Dichloromethane was used as the apolar solvent. The micelle water content for these mixtures can be varied from $w_{0}=2$ to $w_{0}=8$ for CTAB and from $w_{0}=1$ to $w_{0}=4$ for CTAC. At a lower water content the surfactant salt will not dissolve, and at a higher water content phase separation takes place into a turbid phase (the introduction of a cosurfactant can extend the region in which thermodynamically stable reverse micelles form, ${ }^{6,29,30}$ but since cosurfactants usually contain hydroxyl groups of which the vibrational absorptions spectrally overlap with those of the studied water, their use is not desirable in this study). We also studied larger reverse micelles at an elevated temperature of $315 \mathrm{~K}$ using a 1:2 mixture of isooctane and chloroform as a solvent. ${ }^{12}$ For this quaternary mixture, the radius of the water pool was found to increase almost linearly with $w_{0}=\left[\mathrm{H}_{2} \mathrm{O}\right] /[\mathrm{CTAB}]$, according to $r=2.1 w_{0}$ for $w_{0}>10$, as shown by TRFQ experiments. ${ }^{12}$

CTAB and CTAC surfactant salts and apolar solvents were obtained from Aldrich and used without further purification. CTAB micelles in dichloromethane were prepared for $w_{0}=2,4$, and 8. CTAC micelles were prepared for $w_{0}=1,2$, and 4. We used a sample cell of $1 \mathrm{~mm}$ optical path length, and the water volume fraction was kept at 1\%. CTAB micelles in 1:2 mixtures of isooctane and chloroform were prepared for $w_{0}=15$ and 30 . These samples were studied using a variable-temperature cell of $500 \mu \mathrm{m}$ optical path length at $315 \mathrm{~K}$. The water volume fraction for these samples was $3 \%$. All micelle samples contained isotopically diluted water in a volume ratio of 1:30 of $\mathrm{H}_{2} \mathrm{O}$ and $\mathrm{D}_{2} \mathrm{O}$. Bulk halogenic solutions of $\mathrm{NaBr}$ (Aldrich, SigmaUltra grade) of molality 2 and 6 were studied using a cell of $200 \mu \mathrm{m}$ optical path length, using a 1:500 isotopic mixture of $\mathrm{H}_{2} \mathrm{O}$ and $\mathrm{D}_{2} \mathrm{O}$.

We performed ultrafast mid-infrared pump-probe spectroscopy on the $\mathrm{O}-\mathrm{H}$ stretch vibration of diluted HDO in $\mathrm{D}_{2} \mathrm{O}$ confined in the cationic micelles. Isotopically diluted water samples are used to prevent the signals to be affected by intermolecular resonant energy transfer of the $\mathrm{OH}$-stretch vibrations and to limit heating of the sample. In the experiment, a first intense mid-infrared light pulse excites the $\mathrm{OH}$ oscillators, inducing transmission changes for a weak second time-delayed probe pulse. After excitation, the transmission is increased at frequencies matching the $\nu_{\mathrm{OH}}=0 \rightarrow 1$ transition (because of ground state depletion of the $\mathrm{OH}$-stretch vibration and stimulated emission out of the $v=1$ state), while the transmission is decreased at the more redshifted frequencies matching the $\nu_{\mathrm{OH}}=1 \rightarrow 2$ transition (because of absorption due to the induced population in the $v=1$ state).

Our femtosecond mid-infrared light source has been described previously elsewhere. ${ }^{27}$ The generated probe pulses are spectrally broad compared to the pump pulses (200 and $100 \mathrm{~cm}^{-1}$ full width at half maximum, respectively), and the time resolution is $\sim 120 \mathrm{fs}$. After reflecting the probe beam off a glass window to lower its intensity by $95 \%$, the pump and probe beams are overlapped and focused in the sample. The transmitted probe beam is sent through a polarizer and spectrally resolved on a nitrogen-cooled $\mathrm{HgCdTe}$ detector array using a polychromator. Using an automated rotatable mount, the polarizer can be set either parallel or perpendicular with respect to the pump polarization, and at each delay point both these probe components are detected consecutively. In this way, pump induced transmission changes for both the probe component polarized parallel $\left(\Delta \alpha_{\|}\right)$and perpendicular $\left(\Delta \alpha_{\perp}\right)$ with respect to the pump polarization are measured.

The two polarization components are used to construct the so-called rotation-free signal $\Delta \alpha_{\mathrm{RF}}(\omega, t)$ and the anisotropy parameter $R(\omega, t)$ (depending both on the probe frequency $\omega$ and the probe time delay $t$ ) (see the Appendix). The rotation-free signal is given by

$$
\Delta \alpha_{\mathrm{RF}}(\omega, t)=\frac{1}{3}\left(\Delta \alpha_{\|}+2 \Delta \alpha_{\perp}\right)
$$

This signal is not affected by molecular reorientation. The anisotropy parameter is defined as

$$
R(\omega, t)=\frac{\Delta \alpha_{\|}-\Delta \alpha_{\perp}}{\Delta \alpha_{\|}+2 \Delta \alpha_{\perp}}
$$

This parameter reflects the molecular reorientation. 


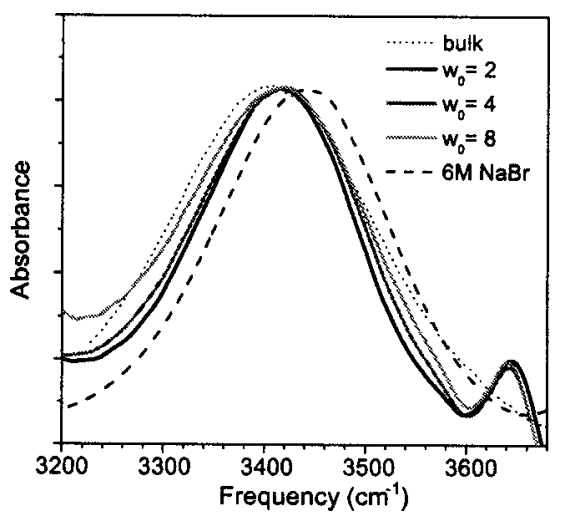

FIG. 1. The $\mathrm{OH}$-stretch absorption of liquid $\mathrm{HDO}: \mathrm{D}_{2} \mathrm{O}$ in $\mathrm{CTAB}$ micelles of various sizes, after subtraction of the $\mathrm{CH}_{2} \mathrm{Cl}_{2}$ solvent background. The $6 \mathrm{M} \mathrm{NaBr}$ spectrum is an absorption spectrum for a solution of $\mathrm{NaBr}$ in 1:500 $\mathrm{H}_{2} \mathrm{O}: \mathrm{D}_{2} \mathrm{O}$ of molality 6 . The bulk spectrum is an absorption spectrum for $1: 1000 \mathrm{H}_{2} \mathrm{O}: \mathrm{D}_{2} \mathrm{O}$.

\section{RESULTS AND DISCUSSION}

\section{A. Linear absorption}

The OH-stretch absorption spectra of water in CTAB reverse micelles of three different sizes are shown in Fig. 1. Since CTAB micelles contain bromide counterions, we compare these spectra with a $6 M \mathrm{NaBr}$ bulk solution, which contains about the same concentration of bromide ions as a $w_{0}=8 \mathrm{CTAB}$ reverse micelle. Anions are known to have an effect on the $\mathrm{OH}$-stretch absorption spectrum of water. ${ }^{31}$ Water in the hydration shell of the bromide ion has an absorption centered at $3475 \pm 11 \mathrm{~cm}^{-1}$, which is blueshifted with respect to the bulk water absorption centered at $3410 \mathrm{~cm}^{-1}$.

The $\mathrm{OH}$-stretch absorption of water in CTAB micelles peaks near $3415 \mathrm{~cm}^{-1}$ for all sizes of micelles (a smaller absorption is observed near $3650 \mathrm{~cm}^{-1}$, which arises from small amounts of HDO dissolved in the dichloromethane solvent). Quite remarkably, only a narrowing of the $\mathrm{OH}$-stretch absorption spectrum is observed with respect to bulk water, and no blueshift due to the bromide counterions. This indicates that the hydration shell of the bromide ion has different properties in reverse micelles than in bulk water.

\section{B. Vibrational relaxation}

\section{Distinguising anion-bound and bulklike water by $T_{1}$}

Figure 2 shows the rotation-free signal recorded for four sizes of micelles at a single absorption frequency in the $\mathrm{OH}$ stretch band. The vibrational relaxation contains a short- and a long-lived component, the short-lived component becoming more pronounced with increasing micelle size. After 3 ps the dynamics becomes dominated by the slower decay, and we will discuss this dynamics first.

The slower vibrational relaxation component has an associated exponential $T_{1}$ time constant of $>4$ ps. Vibrational relaxation on such a long time scale does not occur in bulk $\mathrm{HDO}: \mathrm{D}_{2} \mathrm{O}$. To identify what causes this slower decay, we performed measurements on the same micellar system as $\mathrm{CTAB}$, but with the bromide counterions exchanged for

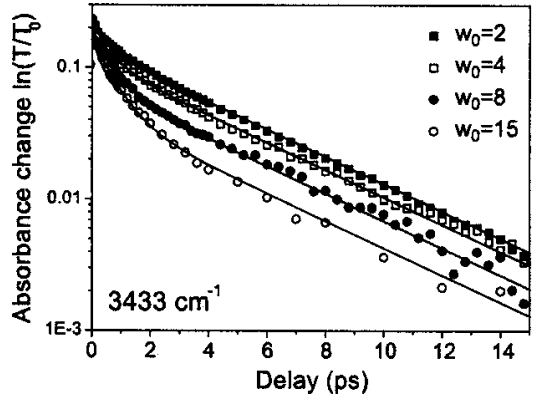

FIG. 2. Pump induced absorbance change plotted as a function of probe delay at $3443 \mathrm{~cm}^{-1}$ at various micelle sizes. The vibrational relaxation has biexponential character. The absorbance change is defined as $\ln \left(T / T_{0}\right)$, where $T$ is the time-dependent transmittance after excitation by the pump and $T_{0}$ the time-independent transmittance in the absence of pump excitation.

chloride ions. This can be accomplished by preparing micelles using the surfactant salt CTAC instead of CTAB. Figure 3 compares the transient absorption change for the $\mathrm{OH}$ stretch vibration of water in CTAC and CTAB reverse micelles of equal size $\left(w_{0}=2\right)$. Clearly, the vibrational relaxation of water in CTAC micelles is faster than in CTAB micelles. Studies on bulk ionic solutions have shown a similar dependence of the vibrational relaxation on the nature of the halogenic anion. ${ }^{32}$ In these studies it was found that water coordinating chloride ions shows a significantly faster vibrational relaxation than water coordinating bromide ions, just as we observe for water in CTAC and CTAB micelles. Therefore, we assign the long-lived component to water solvating the surfactant counterions.

From Fig. 2 we see that within the first 3 ps an initial fast vibrational decay is observed in the transient absorption change. To determine its time constant, we describe the rotation-free signal at each frequency with a fast and a slow contribution. We also measure small absorption changes due to heating of the sample by the intense pump pulse. In a kinetic model we account for the precise dynamics of this thermal signal $\Delta \alpha_{T}$, which is subtracted from the measured parallel and perpendicular transmission changes to obtain the pure pump-probe signals $\Delta \alpha_{\|}(\omega, t)$ and $\Delta \alpha_{\perp}(\omega, t)$ (see the Appendix). We describe the rotation-free signal at each frequency with a fast and a slow contribution.

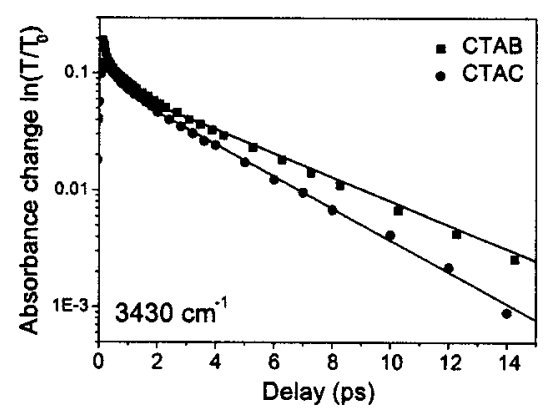

FIG. 3. Pump induced absorbance change plotted as a function of probe delay at $3430 \mathrm{~cm}^{-1}$, comparing CTAB $(\square)$ and CTAC $(\bullet)$ micelles of $w_{0}$ $=2$. The slower component in the vibrational relaxation is associated with the surfactant counterion, which is bromide for $\mathrm{CTAB}$ and chloride for CTAC. Water in the first solvation shell of chloride has a faster vibrational relaxation than the solvation shell water of bromide (Ref. 33). 


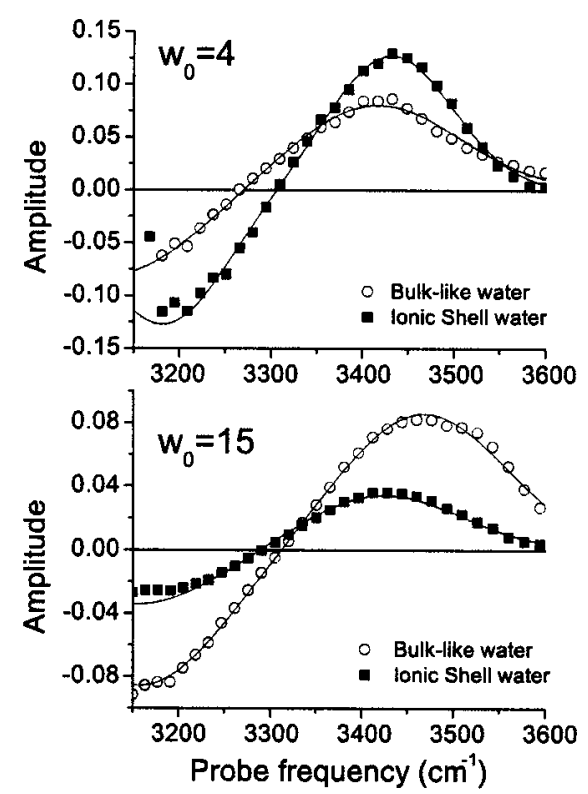

FIG. 4. Spectral pump-probe amplitudes for anion-bound and bulklike $\mathrm{OH}$ groups as a function of probe frequency $\omega$. Following the definitions in Eq. (3), the bulklike water spectrum ( $\mathbf{\square})$ equals $A_{\text {fast }}(\omega)$ and the anion-bound water spectrum (O) equals $A_{\text {slow }}(\omega)$.

$$
\begin{aligned}
& \Delta \alpha_{\mathrm{RF}}(\omega, t)=\Delta \alpha_{\mathrm{RF}, \text { fast }}+\Delta \alpha_{\mathrm{RF}, \text { slow }}, \\
& \Delta \alpha_{\mathrm{RF}, \text { fast }}(\omega, t)=A_{\text {fast }}(\omega) e^{-t / T_{\text {fast }},} \\
& \Delta \alpha_{\mathrm{RF}, \text { slow }}(\omega, t)=A_{\text {slow }}(\omega) e^{-t / T_{\text {slow }}} .
\end{aligned}
$$

We find a fast component of $0.7 \pm 0.2 \mathrm{ps}$ and a slower anion solvation shell component of $4.1 \pm 0.2 \mathrm{ps}(3.4 \pm 0.2 \mathrm{ps}$ for CTAC) for all sizes of micelle. The obtained spectra $A_{\text {fast }}(\omega)$ and $A_{\text {slow }}(\omega)$ are shown in Fig. 4 . The time scale of the fast component is very similar to the vibrational relaxation rate observed for bulk $\mathrm{HDO}: \mathrm{D}_{2} \mathrm{O}$, indicating that $\mathrm{OH}$ oscillators hydrogen bonded to other water molecules cause this decay. The relative fraction of the fast component increases as we increase the size of the micelle, as displayed in Fig. 6. This trend can be well understood, since larger micelles contain less bromide ions, and therefore more water outside the bromide solvation shells.

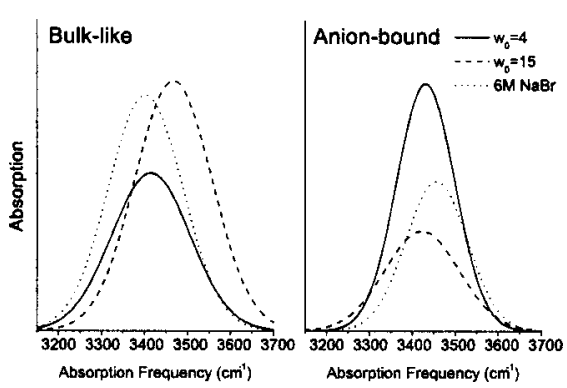

FIG. 5. Gaussian absorption line shapes for bulklike and anion-bound water, extracted from pump-probe spectra. The data are scaled relative to the sum of the spectrally integrated peak area of bulklike and anion-bound water.

\section{Absorption line shapes anion-bound and bulklike water}

For each micelle both the spectra $A_{\text {slow }}(\omega)$ and $A_{\text {fast }}(\omega)$ were fitted using two Gaussian line shapes, one positive Gaussian of center frequency $\nu$ and width $\Delta$ to represent the $\nu_{\mathrm{OH}}=0 \rightarrow 1$ bleaching part of the pump-probe spectrum, and one negative Gaussian of equal shape but redshifted in frequency by an anharmonicity $\alpha$ to represent the $\nu_{\mathrm{OH}}=1 \rightarrow 2$ induced absorption. The line shape parameters are listed in Table I. In Fig. 5 the obtained absorption line shapes are shown for two sizes of micelles and for a $6 M \mathrm{NaBr}$ solution.

As seen from the right panel in Fig. 5, the hydration shell of $\mathrm{Br}^{-}$absorbs more to the red in CTAB micelles than in bulk water. This may result from a different solvating structure of bromide ions in micelles. The absorption line shape for bulklike water in micelles is also different compared to bulk water. For micelles of $w_{0} \geqslant 8$, the bulklike water has a blueshifted absorption frequency. This points to weakened hydrogen bonding in the core of the micelles, as observed earlier for water in anionic micelles. ${ }^{27}$ For the smallest micelles $\left(w_{0}=2,4\right)$ the bulklike water absorption is also slightly blueshifted with respect to bulk water, however, much less than for the larger micelles. The water molecules corresponding to this absorption likely coordinate the cationic head groups of the $\mathrm{CTA}^{+}$surfactant, resulting in an increase of the hydrogen bonding to other molecules. As a result, the absorption is less blueshifted than that of water molecules in the core of larger micelles.

\section{Inhomogeneous distribution of counterions}

For bulk ionic solutions it was found that the relaxation rate of anion-bound water increases with anion

TABLE I. OH-stretch vibrational and reorientational lifetimes (in ps) and parameters of bulklike and anionbound water in CTAB reverse micelles.

\begin{tabular}{cccccccc}
\hline \hline$w_{0}$ & $T_{1, \text { bulk }}(\mathrm{ps})$ & $T_{1, \text { anion }}(\mathrm{ps})$ & $\tau_{\text {bulk }}(\mathrm{ps})$ & $\tau_{\text {anion }}(\mathrm{ps})$ & $R_{0}$ & $Q^{2}$ & $\theta(\mathrm{deg})$ \\
\hline 2 & $1.2(0.2)$ & $4.4(0.2)$ & $3(1)$ & $55(10)$ & $0.28(0.02)$ & $0.8(0.1)$ & $25(5)$ \\
4 & $0.7(0.2)$ & $4.2(0.2)$ & $3(1)$ & $17(2)$ & $0.29(0.02)$ & $0.8(0.1)$ & $25(5)$ \\
8 & $0.7(0.1)$ & $4.3(0.3)$ & $3(1)$ & $9(2)$ & $0.28(0.02)$ & $0.8(0.1)$ & $25(5)$ \\
15 & $0.7(0.1)$ & $4.0(0.3)$ & $3(1)$ & $8(2)$ & $0.28(0.02)$ & $0.8(0.2)$ & $25(10)$ \\
& & $\ldots$ & $2.5(0.2)$ & $\ldots$ & $0.3(0.02)$ & $\ldots$ & $\ldots$ \\
$\mathrm{Bulk}^{\mathrm{a}}$ & $0.7(0.1)$ & $\ldots$ & $3(1)$ & $9(2)$ & $0.26(0.02)$ & $0.8(0.2)$ & $25(10)$ \\
$2 M \mathrm{NaBr}$ & $0.6(0.1)$ & $2.5(0.2)$ & $3(1)$ & $9(2)$ & $0.26(0.02)$ & $0.8(0.1)$ & $25(5)$ \\
$6 M \mathrm{NaBr}$ & $0.7(0.1)$ & $3.0(0.2)$ & $3(5)$ & & & &
\end{tabular}

${ }^{\mathrm{a}}$ Values taken from Rezus and Bakker (Ref. 44). 
concentration. ${ }^{33}$ Using these previous results as a reference, we can use the measured vibrational relaxation rates in the reverse micelles to determine the local concentration of bromide ions. The relaxation of anion-bound water in the micelles is remarkably slow $(4.1 \mathrm{ps})$, which implies that a very high local concentration of $\mathrm{Br}^{-}$must be present. To compare, a nearly saturated solution of $10 M \mathrm{NaBr}$ in water gives rise to a vibrational relaxation rate of anion-bound water of only 3.6 ps. In reverse micelles even denser regions of bromide ions must occur, at molarities that cannot be reached in bulk solutions.

Interestingly, the vibrational relaxation of anion-bound water is anomalously slow for all studied sizes of micelles, as shown in Fig. 2. For all micelles the $T_{1}$ times are $>3.6 \mathrm{ps}$, showing that the local bromide concentration must be higher than $10 M$ in each case. This is quite remarkable, as the average bromide concentration per micelle decreases from $18 M$ to $3.5 M$ when increasing the micelle size from $w_{0}=2$ to $w_{0}$ $=15$. The data thus show that the bromide ions are strongly inhomogeneously distributed over the micelles. The large value of $T_{1}$ found for all micelles indicates that a very high density of ions must be present in a restricted region at the micellar interface. We thereby show that most anions are located inside or directly adjacent to the Stern layer.

These observations are consistent with a considerable amount of experimental $^{8,14,30,34,35}$ and theoretical ${ }^{9,36-38}$ work which has been done on ion binding at charged aqueous interfaces of normal and reversed micelles. The degree of counterion binding at the interface is often expressed by the parameter $\beta$, giving the number of counterions that are bound per surfactant molecule. Experimental estimates of $\beta$ exist for normal micelles dispersed in water ${ }^{9,34,35}$ and for large water-in-oil microemulsions, ${ }^{14,30}$ obtained by small angle neutron scattering, ${ }^{34}$ small angle x-ray scattering, ${ }^{35}$ NMR, ${ }^{14}$ and chemical trapping techniques. ${ }^{30}$ For most surfactant salts, including $\mathrm{CTAB},{ }^{30,34} \beta$ is in the range of $0.6-$ 0.9 . This means that for normal micelles and large reverse micelles $\left(w_{0} \rightarrow \infty\right)$, the majority of the counterions remain associated with the surfactant molecules within the Stern layer and do not enter the bulk aqueous phase. Experimental data on the counterion distributions within small reverse micelles are, however, very limited.

Our results show that also in small cationic reverse micelles, the counterion distribution is very inhomogeneous over the micelle, with most bromide ions concentrated at the micelle interface. For small anionic (AOT) reverse micelles extensive MD simulations exist, ${ }^{37,38}$ which also suggest that the counterions mainly associate with the surfactant head groups. We may state that the anion-bound water component observed in the relaxation is mainly due to interfacial water, located in or near the Stern layer of the CTAB reverse micelle.

\section{Degree of counterion solvation}

By spectrally integrating the obtained line shapes for bulklike and anion-bound water (see Fig. 5), we obtain the relative fractions $A_{\text {bulk }}$ and $A_{\text {anion }}$ for bulklike and anionbound water within the reverse micelles. Since we know the molar ratio between water and counterions, from these frac-

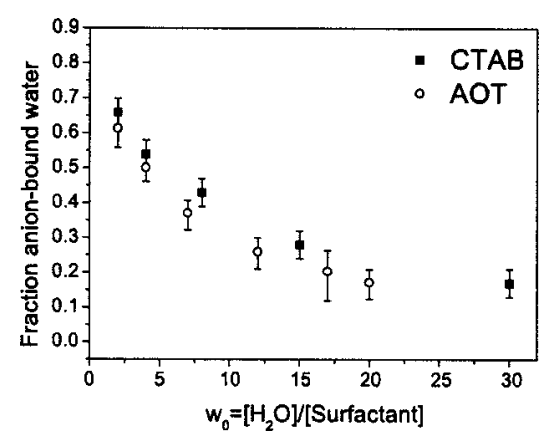

FIG. 6. Relative fraction anion-bound water $A_{\text {anion }}$ (see Table II) obtained by spectrally integrating the peak areas of bulklike and anion-bound water (see Fig. 5). For comparison, the interfacial water fractions for anionic AOT micelles (Ref. 27) are shown as well.

tions we can deduce the average counterion hydration number. Note that no significant absorption cross-section difference is expected between anion-bound and bulklike water. ${ }^{31}$

While the bromide solvation shell contains six water molecules in bulk water, ${ }^{31,39}$ the average hydration number in micelles turns out lower for the smallest micelles $w_{0}=2$ and 4 , for which we find a hydration number of only $2.6 \pm 0.2$ and $4.3 \pm 0.4$, respectively, which means that the bromide anions are only partly hydrated. The hydration number increases up to $7 \pm 2$ for $w_{0}>15$, which is close to the bulk anion hydration number of 6 . Since the $\mathrm{Br}^{-}$anions reside predominantly at the interface, it can thus be concluded that the interface hydration increases as soon as the water content of the micelles is increased, as was shown previously for anionic reverse micelles. ${ }^{14,27,28}$ The increased hydration of the interfacial region implies a lowering of the local concentration of $\mathrm{Br}^{-}$anions. This lowering in concentration does not lead to a significant decrease of $T_{1}$, probably because in this concentration regime all donated hydrogen bonds are to $\mathrm{Br}^{-}$, and not to other water molecules. It was previously shown that the concentration dependence of $T_{1}$ of the solvating water molecules can be well explained from the increasing fraction of donated hydrogen bonds to other water molecules when the anion concentration is lowered. ${ }^{33}$

The relative amplitude of the $\mathrm{Br}^{-}$hydration shell component matches quite well with the interfacial water fraction of anionic AOT micelles, as shown in Fig. 6. Water molecules that solvate the sulfonate anionic head group of the AOT surfactant show a longer vibrational lifetime $(2.8 \mathrm{ps})$ than water molecules coordinating other water molecules. On the basis of this long lifetime, such interfacial molecules can be distinguished from water in the core of the micelles, just as we can distinguish water inside and outside the bromide hydration shells in the CTAB system. As opposed to CTAB reverse micelles, which contain free bromide anions, the anionic group of the AOT is covalently bonded to the hydrophobic tail that forms the interface with the apolar solvent outside the micelle. The anionic head group is thereby geometrically confined to the outer shell of the micelle. Both the sulfonate head group $^{40}$ and the bromide anion ${ }^{31,39}$ can hydrogen bond six $\mathrm{OH}$ groups, and no large cross-sectional difference exist for various anionic groups. ${ }^{31}$ The degree of hydration of $\mathrm{Br}^{-}$in $\mathrm{CTAB}$ reverse micelles shows therefore 
TABLE II. Relative fractions and line shape parameters of bulklike and anion-bound water in CTAB reverse micelles.

\begin{tabular}{|c|c|c|c|c|c|c|c|c|c|}
\hline$w_{0}$ & Solv $^{\mathrm{a}}$ & $\begin{array}{c}T \\
(\mathrm{~K})\end{array}$ & $A_{\text {bulk }}$ & $A_{\text {anion }}$ & $n_{\mathrm{Br}^{-}}{ }^{\mathrm{b}}$ & $\begin{array}{c}\nu_{\text {bulk }} \\
\left(\mathrm{cm}^{-1}\right)\end{array}$ & $\begin{array}{c}\nu_{\text {anion }} \\
\left(\mathrm{cm}^{-1}\right)\end{array}$ & $\begin{array}{c}\Delta_{\text {bulk }} \\
\left(\mathrm{cm}^{-1}\right)\end{array}$ & $\begin{array}{r}\Delta_{\text {anion }} \\
\left(\mathrm{cm}^{-1}\right)\end{array}$ \\
\hline 2 & I & $295(2)$ & $0.34(0.05)$ & $0.66(0.05)$ & $2.6(0.2)$ & 3409 (5) & $3433(5)$ & $69(8)$ & $61(8)$ \\
\hline 4 & I & 295 (2) & $0.46(0.05)$ & $0.54(0.05)$ & $4.3(0.4)$ & $3415(5)$ & $3431(5)$ & $92(8)$ & $68(8)$ \\
\hline 8 & I & $295(2)$ & $0.57(0.05)$ & $0.43(0.05)$ & $6.9(0.8)$ & $3463(5)$ & $3426(5)$ & $80(8)$ & $68(8)$ \\
\hline 15 & II & $315(5)$ & $0.7(0.1)$ & $0.3(0.1)$ & $7(2)$ & $3467(5)$ & $3422(5)$ & $90(8)$ & $87(8)$ \\
\hline 30 & II & $315(5)$ & $0.8(0.1)$ & $0.2(0.1)$ & $7(2)$ & $3501(5)$ & $3437(5)$ & $81(8)$ & $64(8)$ \\
\hline Bulk & III & 298 (2) & 1 & 0 & $\cdots$ & $3419(5)$ & $\cdots$ & $92(8)$ & $\cdots$ \\
\hline $2 M \mathrm{NaBr}$ & IV & 298 (2) & $0.82(0.05)$ & $0.18(0.05)$ & $6(1)$ & $3400(5)$ & $3450(5)$ & $102(8)$ & $73(8)$ \\
\hline $6 M \mathrm{NaBr}$ & IV & 298 (2) & $0.65(0.05)$ & $0.35(0.05)$ & $4.9(0.6)$ & $3402(5)$ & $3456(5)$ & $86(8)$ & $72(8)$ \\
\hline
\end{tabular}

${ }^{a}$ Solvents used: I, dichloromethane; II, 1:2 isooctane:chloroform; III, 1:1000 $\mathrm{H}_{2} \mathrm{O}: \mathrm{D}_{2} ; \mathrm{IV}, 1: 500 \mathrm{H}_{2} \mathrm{O}: \mathrm{D}_{2} \mathrm{O}$.

${ }^{\mathrm{b}}$ Bromide coordination number has been determined using a 1:1 cross-sectional ratio between bulk and anion-bound water.

the same dependence on the water content $w_{0}$ as does the degree of hydration of sulfonate in AOT micelles.

\section{Orientational relaxation}

Figure 7 shows anisotropy decay curves for three sizes of micelles.

The initial anisotropy $R(\omega, 0)$ equals $0.29 \pm 0.02$ at all probe frequencies $\omega$, below the limiting value of 0.4 . This indicates that our measurement does not capture all of the molecular reorientational dynamics, and a fast anisotropy decay occurs within the 100 fs time resolution of our experiment. A fast orientational relaxation within the first 150 fs has been experimentally resolved for bulk $\mathrm{HDO}: \mathrm{D}_{2} \mathrm{O}{ }^{41,42}$ This decay has been assigned to nondiffusive librational motions of water, which are equally present in the aqueous interior of reverse micelles. ${ }^{24}$

From 0 to 2 ps an initial fast decay is observed in the anisotropy curves. Within this time window, the short-lived signal of bulklike water molecules contributes to the anisotropy. The fast decay is on the order of $2.5 \mathrm{ps}$, similar to the orientational relaxation found for bulk water. The fast component in the anisotropy decay becomes more pronounced for larger micelles, where the bulklike water fraction is also larger. This suggests that bulklike water is at least partially responsible for the fast anisotropy decay.

After $\sim 2$ ps only bromide solvation shell water contributes to the signal because of its much longer vibrational lifetime than bulklike water. We observe a monoexponential de-

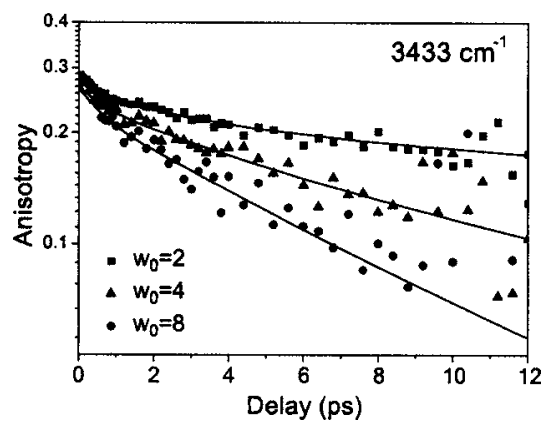

FIG. 7. Anisotropy decay of the $\mathrm{OH}$-stretch vibration at $3433 \mathrm{~cm}^{-1}$ for three sizes of CTAB reverse micelles $\left(w_{0}=2,4\right.$, and 8$)$. The mobility of anionbound water increases when the micelle water content is increased. cay which is much faster for micelles with a high water content than for micelles with a low water content. This shows that the mobility of the anion-bound water gets considerably lowered when the confinement is increased.

In analogy with the description of the vibrational relaxation, we will allow for different orientational mobilities for bulklike and anion-bound water in describing the anisotropy decay. By use of Eq. (3), we can rewrite the expression for the anisotropy in Eq. (2) as follows:

$$
\begin{aligned}
& R(\omega, t)=C_{\text {bulk }} R_{\text {bulk }}(t)+C_{\text {anion }} R_{\text {anion }}(t), \\
& C_{\text {bulk }}(\omega, t)=\frac{\Delta \alpha_{\mathrm{RF}, \text { fast }}}{\Delta \alpha_{\mathrm{RF}}}, \quad C_{\text {anion }}(\omega, t)=\frac{\Delta \alpha_{\mathrm{RF}, \text { slow }}}{\Delta \alpha_{\mathrm{RF}}} .
\end{aligned}
$$

$R_{\text {bulk }}(t)$ and $R_{\text {anion }}(t)$ are the anisotropy parameters for the bulk and anion-bound water subensembles. The timedependent fractions $C_{\text {bulk }}$ and $C_{\text {anion }}$ follow directly from the fit to the rotation-free signal. We model $R_{\text {bulk }}$ to decay single exponentially with an associated decay constant of $\tau_{\text {bulk }}$. $R_{\text {anion }}$ is modeled to decay biexponentially, one fraction having a relative amplitude $Q^{2}$ and an associated decay constant $\tau_{\text {anion }}$, and a second fraction having a faster associated decay constant $\tau^{\prime}$.

$$
\begin{aligned}
& R_{\text {bulk }}(t)=R_{0}\left(e^{-t / \tau_{\text {bulk }}}\right), \\
& R_{\text {anion }}(t)=R_{0}\left(Q^{2} e^{-t / \tau_{\text {anion }}}+\left(1-Q^{2}\right) e^{-t / \tau^{\prime}}\right) .
\end{aligned}
$$

This model corresponds to the physical situation in which slowly reorienting anion-bound water-mostly located at the micelle interface - can undergo a second faster reorientation within a cone of semiangle $\theta$ on a time scale $\tau_{\text {cone }}{ }^{24,43}$ The relative amplitude $Q^{2}$ of the slow decay in the anion-bound water reorientation can be related to the cone semiangle by

$$
Q^{2}=\left[\frac{1}{2} \cos \theta(1+\cos \theta)\right]^{2} \text {. }
$$

Note that the case $Q^{2}=1$ corresponds to the case of a cone angle of $0^{\circ}$, in which the faster reorientation component for the anion-bound water is effectively put to zero. Values of $Q^{2}$ smaller than 1 imply a larger contribution of fast reorientation for the anion-bound water. The fast component in the anion orientational relaxation $\tau^{\prime}$ is related to the time constant $\tau_{\text {cone }}$ by 


$$
\tau^{\prime}=1 /\left(\tau_{\text {cone }}^{-1}+\tau_{\text {anion }}^{-1}\right)
$$

This time constant will in practice be nearly equal to $\tau_{\text {cone }}$ because for the cases considered $\tau_{\text {anion }} \gg \tau_{\text {cone }}$.

Having determined the time-dependent fractions $C_{\text {bulk }}$ and $C_{\text {anion }}$ from the vibrational relaxation data, the model contains five remaining adjustable parameters: $Q^{2}, R(\omega, 0)$ $\equiv R_{0}, \tau_{\text {cone }}, \tau_{\text {bulk }}$, and $\tau_{\text {anion }}$, of which only $\tau_{\text {anion }}$ is allowed to vary with micelle size. The values for these parameters are obtained from a global fit to the anisotropy curves at all spectral frequencies between 3350 and $3500 \mathrm{~cm}^{-1}$ for all micelles, simultaneously. The obtained fitting parameters are summarized in Table I.

From the obtained value for $\tau_{\text {bulk }}$ of $3 \mathrm{ps,} \mathrm{we} \mathrm{conclude}$ that within our experimental accuracy, the reorientation of water outside the anion solvation shells is equally fast as the reorientation known for bulk $\mathrm{HDO}: \mathrm{D}_{2} \mathrm{O}$ [2.6 ps (Ref. 44)]. This water is mainly located in the core of the droplets, and its dynamics does not seem to be strongly affected by the confinement, as was observed previously for anionic reverse micelles. $^{27}$

The reorientation rate of anion-bound water is observed to strongly decrease upon confinement, as seen from the values determined for $\tau_{\text {anion. }}$. An increasing hydration of the Stern layer of the reverse micelle apparently increases the mobility of the interfacial water. At a water content of $w_{0}$ $=8$, the reorientation of anion-bound water becomes equal to that of the anion solvation shell in bulk $\mathrm{NaBr}$ solutions, for which the reorientation of bromide solvation shells was shown to occur on a time scale of $11 \mathrm{ps}^{45}$ The average bromide hydration number then equals nearly 6 , just like the bulk hydration number, as seen from Table I.

The time scale of the faster motion of the anion-bound water turns out to be similar to that of the bulklike water. From the fit we find that $\tau_{\text {cone }}$ and $\tau_{\text {bulk }}$ are both in the range $2.5-3.5$ ps. In fact, the quality of the fit is not affected by taking the cone reorientation time constant equal to the bulk reorientation time constant, and within our experimental accuracy we can therefore assume

$$
\tau_{\text {cone }} \equiv \tau_{\text {bulk }} .
$$

The relative amplitude $Q^{2}$ of the slow anisotropy decay is determined to be $0.75 \pm 0.05$, which can be related by Eq. (7) to the average semiangle of a cone in which this restricted motion takes place. We find a cone with a semiangle of $25^{\circ}$. Water at the interfacial Stern layer thus undergoes a restricted reorientation over $50^{\circ}$ at the same rate as adjoining bulklike molecules. Reorientation over larger angles in which the solvating structure of bromide needs to rearrange dramatically or break up occurs on a slower time scale given by $\tau_{\text {anion. }}$. For bulk ionic solutions of $\mathrm{NaBr}$, similar $Q^{2}$ values need to be assumed as well.

A recovery of the anisotropy (occurring if $Q^{2} \sim 1$, see Fig. 8), was recently observed for water in anionic AOT reverse micelles. ${ }^{27}$ Such a dynamic signature is a good indicator of inhomogeneous reorientation of water inside reverse micelles. $^{26,27}$ In the most simple case of a bimodal system, such behavior should be observed when the more slowly reorienting species also has a longer vibrational lifetime than

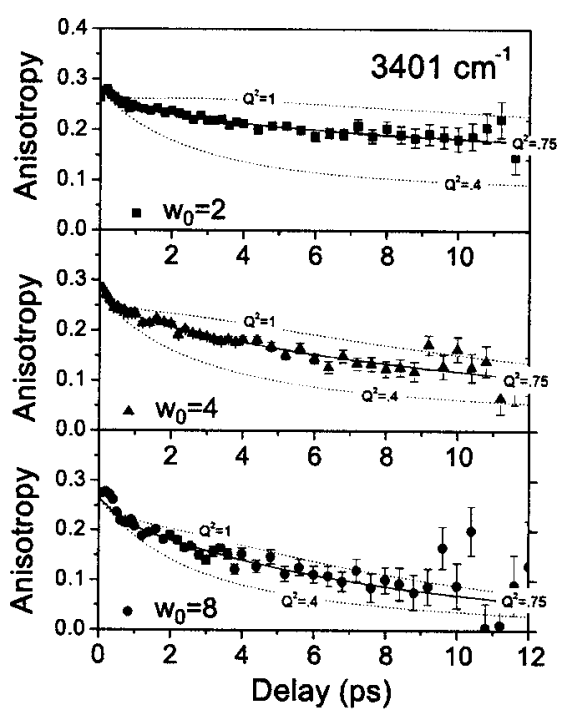

FIG. 8. Anisotropy decay curves and their fits on a linear scale for three sizes of micelles. For each graph, additional curves for $Q^{2}=1$ and $Q^{2}=0.4$ have been added for comparison, corresponding to cone angles of $\theta=0^{\circ}$ and $43^{\circ}$, respectively. For $Q^{2}$ very near to 1 , the anisotropy decay is slowed down at early delays, in specific cases leading even to a recovery of the anisotropy.

the faster reorienting species, as is the case for water in CTAB micelles. Figure 8 illustrates, however, that a certain degree of faster motion for the slowly reorienting species can strongly suppress this characteristic dynamics for inhomogeneous reorientation, even though the water reorientation throughout the droplet remains strongly inhomogeneous.

The fact that an anisotropy recovery is observed in AOT reverse micelles and not in CTAB reverse micelles can be explained by differences between the anion-bound water found in $C T A B$ micelles and the interfacial water found in AOT micelles. Interfacial water in AOT micelles consists of water molecules hydrogen bonding to the anionic sulfonate head groups of the AOT, which are rigidly constricted to the outer shell of the micelle. Anion-bound water in CTAB micelles also has interfacial character, since most anions are located near or inside an interfacial Stern layer. The hydrogen bonding of water to sulfonate and bromide is, however, strikingly different, as illustrated in Fig. 9. The bromide ion is much larger than the oxygens of sulfonate that can accept hydrogen bonds from water. Because of the large polarizable electron cloud of the bromide ion, its hydrogen bonding will be much less directional than in the case of sulfonate. A less directional hydrogen bond will allow for a higher orientational mobility of the neighboring water molecules. This can explain a $Q^{2}$ value closer to unity for water hydrogen bonding to AOT, as compared to water hydrogen bonding to halogenic ions.

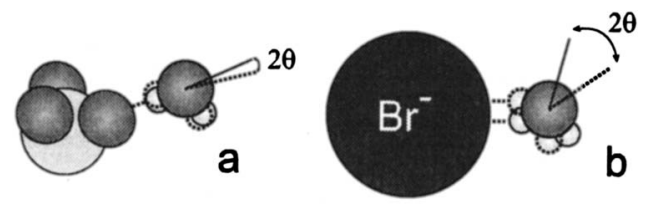

FIG. 9. Directionality of the hydrogen-bond between water and sulfonate (a) and between water and bromide (b). The angle $\theta$ refers to the cone semiangle given in Eq. (7). 


\section{CONCLUSIONS}

We studied the ultrafast dynamics of isotopically diluted water confined in cationic CTAB reverse micelles. Based upon differences in the vibrational relaxation rate, we can distinguish between $\mathrm{OH}$ groups that are hydrogen bonded to surfactant counterions and $\mathrm{OH}$ groups that are not. We find an anomalously slow vibrational relaxation rate for water hydrogen bonded to the counterions. This observation implies that very high local concentrations of $\mathrm{Br}^{-}$anions $(>10 M)$ must be present, and that the $\mathrm{Br}^{-}$anions are not homogeneously distributed over the micelle. Together with the observation that the vibrational relaxation rate of anionbound water does not vary significantly with the micelle water content (and therefore neither does the local $\mathrm{Br}^{-}$concentration), we conclude that most of the counterions reside at the micellar interface in or near the Stern layer. Anion-bound water therefore has strong interfacial character.

Within our experimental accuracy, bulklike water in the core of the micelles is found to have an orientational mobility similar to bulk liquid water, as was found previously for anionic AOT micelles. Water bound to $\mathrm{Br}^{-}$, found predominantly at the micellar interface, has a much lower orientational mobility. Its associated reorientational time constant strongly decreases with micelle size from 55 to $\sim 8 \mathrm{ps}$. This decrease shows that water molecules in the bromide-rich interfacial region of the micelle become more mobile when the micelle water content is increased.

Besides a slow reorientational motion, anion-bound water molecules also undergo a faster but restricted reorientation on the same time scale as the bulklike molecules. This motion can on average be described by diffusional motion in a cone with a semiangle of $25^{\circ}$. The size of the cone is independent of the micelle size. This fast reorientational motion was not observed for interfacial water in AOT micelles. ${ }^{27}$ For AOT micelles, the interfacial water is characterized by its hydrogen bonding to the anionic sulfonate head groups of the AOT surfactant molecules. We explain these different mobilities from a lower directionality of the waterbromide hydrogen bond compared to the water-sulfonate hydrogen bond. A hydrogen bond of low directionality allows for a higher mobility of the water molecule while leaving its hydrogen bond intact. Interfacial water in CTAB micelles therefore has a higher orientational mobility than interfacial water in AOT micelles.

\section{ACKNOWLEDGMENTS}

This work is part of the research program of the Stichting voor Fundamenteel Onderzoek der Materie (FOM), which is financially supported by the Nederlandse Organisatie voor Wetenschappelijk Onderzoek (NWO).

\section{APPENDIX: KINETIC MODELING}

We describe our system by two subensembles of water molecules, denoted by the index $i \in\{$ fast, slow $\}$. One group of molecules hydrogen bonds to other water molecules and has a vibrational lifetime $T_{\text {fast }}$. A second group of molecules hydrogen bonds to $\mathrm{Br}^{-}$ions and has a vibrational lifetime $T_{\text {slow. }}$. The population dynamics of excited molecules of each

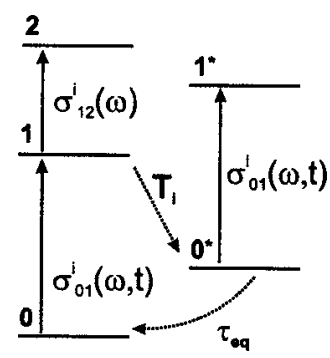

FIG. 10. Schematic overview of the model that describes the population dynamics for both types $i$ of excited HDO molecules ( $i \in\{$ fast, slow $\})$. Relaxation from the excited 1 state proceeds via an intermediate level $0^{*}$. The cross section of the intermediate level is taken to be equal to that of the ground state. The cross section of the $1 \rightarrow 2$ transition is assumed to be time independent.

of these types is schematically depicted in Fig. 10. As was previously found for bulk $\mathrm{HDO}: \mathrm{D}_{2} \mathrm{O},{ }^{44}$ we assume that the vibrational relaxation of the $\mathrm{OH}$ vibration proceeds via an intermediate level $0^{*}$. The excitation subsequently relaxes back to the ground state 0 , thereby thermally populating low frequency modes. The following equations describe the dynamics of the molecules that were excited by the pump pulse:

$$
\begin{aligned}
& \frac{d N_{1, i}}{d t}=-\frac{1}{T_{i}} N_{1, i}, \quad i \in\{\text { fast, slow }\} \\
& \frac{d N_{0, i}^{*}}{d t}=\frac{1}{T_{i}} N_{1, i}-\frac{1}{\tau_{\mathrm{eq}}} N_{0, i}^{*}, \\
& \frac{d N_{0, i}}{d t}=\frac{1}{\tau_{\mathrm{eq}}} N_{0, i}^{*} .
\end{aligned}
$$

$N_{1, i}, N_{0, i}^{*}$, and $N_{0, i}$ refer to the populations of the levels depicted in Fig. 10, for each subensemble $i$. As a boundary condition we assume that on $t=0$ the number of molecules in the excited state equals the number of molecules removed from the ground state:

$$
N_{0, i}(0)=-N_{1, i}(0) \text {. }
$$

When the excited molecules relax to the ground state, the reverse micelles in the sample are gradually heated. Heating affects all water molecules in the reverse micelles, including those molecules that were not excited by the pump pulse. The micelles subsequently cool to the surrounding solvent on a picosecond time scale. ${ }^{19}$ Because of heating, the two crosssection spectra $\left[\sigma_{01}^{i}(\omega, t), i \in\{\right.$ fast, slow $\left.\}\right]$ slightly blueshift and decrease amplitude, resulting in thermal absorption changes $\Delta \alpha_{T}(\omega, t)$ observed in the experiments.

We assume the change of each spectrum $\sigma_{01}^{i}(\omega, t)$ upon heating to be proportional to the total number of molecules $\Sigma_{i} N_{\text {rel }, i}$ that have decayed to the ground state (where $N_{\text {rel }, i}$ is the number of relaxed molecules of type $i$ ), multiplied by an exponential factor to account for the cooling of the micelles to their surroundings: 


$$
\begin{aligned}
& N_{\mathrm{rel}, i}(t)=\int_{t^{\prime}=0}^{t} \frac{1}{\tau_{\mathrm{eq}}} N_{0, i}^{*}\left(t^{\prime}\right) d t^{\prime} \\
& \Delta \sigma_{01}^{i}(\omega, t)=\sigma_{01}^{i}(\omega, t)-\sigma_{01}^{i}(\omega, 0) \propto \sum_{i} N_{\mathrm{rel}, i}(t) \times e^{-t / \tau_{\mathrm{cool}}}
\end{aligned}
$$

Although heated micelles in general show nonexponential cooling behavior, ${ }^{19}$ single-exponential cooling is a good approximation for the time window $(0-40 \mathrm{ps})$ considered in our experiments.

The total rotation-free pump-probe signal can be written in terms of the populations of the levels in Fig. 10, the absorption cross sections $\sigma$ between these levels, and the total concentration of absorbing molecules $C$.

$$
\begin{aligned}
\Delta \alpha(\omega, t)= & \sum_{i}\left[C \Delta \sigma_{01}^{i}(\omega, t)\right. \\
& +N_{1, i}(t) \sigma_{12}^{i}(\omega)+\left(N_{0, i}(t)-N_{1, i}(t)\right. \\
& \left.\left.+N_{0, i}^{*}(t)\right) \sigma_{01}^{i}(\omega, t)\right] .
\end{aligned}
$$

By solving for the rate equations (A1) and assuming that the fraction of excited molecules $\sum_{i} N_{1, i}(0) / C$ is a small number, we find

$$
\begin{aligned}
\Delta \alpha(\omega, t)= & \sum_{i}\left[C \Delta \sigma_{01}^{i}(\omega, t)+N_{1, i}(0)\left[\sigma_{12}^{i}(\omega)\right.\right. \\
& \left.\left.-2 \sigma_{01}^{i}(\omega, 0)\right] e^{-t / T_{i}}\right] .
\end{aligned}
$$

By direct comparison with Eq. (3) we find for the pumpprobe spectrum of each component $i$

$$
A_{i}(\omega)=N_{1, i}(0)\left[\sigma_{12}^{i}(\omega)-2 \sigma_{01}^{i}(\omega, 0)\right]
$$

and for the functional form of the thermal signal

$$
\Delta \alpha_{T}(\omega, t)=C \sum_{i} \Delta \sigma_{01}^{i}(\omega, t)
$$

The number of relaxed molecules $N_{\text {rel }, i}(t)$ determines the dynamics of the thermal signal and has a solution

$$
\begin{aligned}
N_{\mathrm{rel}, i}(t) & =N_{0, i}(t)-N_{0, i}(0) \\
& =N_{1, i}(0)\left(\frac{\tau_{\mathrm{eq}}}{T_{i}-\tau_{\mathrm{eq}}} e^{-t / \tau_{\mathrm{eq}}}-\frac{T_{i}}{T_{i}-\tau_{\mathrm{eq}}} e^{-t / T_{i}}+1\right)
\end{aligned}
$$

From this equation, we see that the dynamics of the total heating signal depends on the initial populations $N_{1, i}(0)$. In our fitting procedure we assume that the area of the positive bleaching part of the spectra $A_{i}(\omega)$ is proportional to these initial populations:

$$
N_{1, i}(0) \propto \int_{\text {bleach }} A_{i}(\omega) d \omega .
$$

In terms of the line shape functions defined in Sec. III B 2 we can write for this integral

$$
\int_{\text {bleach }} A_{i}(\omega) d \omega=N_{1, i}(0) \sigma_{01}^{i}\left(\omega_{\max }, 0\right) \operatorname{Erf}\left(\frac{\alpha_{i}}{2 \sqrt{2} \Delta_{i}}\right),
$$

showing that the proportionality in Eq. (A9) holds when the linewidths $\Delta$, cross sections, and anharmonicities $\alpha$ of the two subensembles $i$ are similar. Considering the $\mathrm{OH}$-stretch line shape for pure HDO: $\mathrm{D}_{2} \mathrm{O}$ and $\mathrm{Br}^{-}$-bound water, ${ }^{31}$ this is a reasonable assumption. We define

$$
f_{i} \equiv N_{1, i}(0) / \sum_{i} N_{1, i}(0)
$$

as the normalized fraction of molecules of type $i$ contributing to the pump-probe signal.

By use of Eqs. (A3) and (A8), we can then rewrite Eq. (A5) for the total pump-probe signal:

$$
\begin{aligned}
\Delta \alpha_{T}(\omega, t)= & \Delta \alpha_{T}(\omega) e^{-t / T_{\text {cool }}} \\
& \times \sum_{i} f_{i}\left(\frac{\tau_{\mathrm{eq}}}{T_{i}-\tau_{\mathrm{eq}}} e^{-t / \tau_{\mathrm{eq}}}-\frac{T_{i}}{T_{i}-\tau_{\mathrm{eq}}} e^{-t / T_{i}}+1\right) .
\end{aligned}
$$

In this equation $\Delta \alpha_{T}(\omega)$ is the proportionality constant between $\Delta \alpha_{T}(\omega, t)$ and $\sum_{i} N_{0, i}^{\prime}(t)$. This factor equals the thermal spectrum found at large delays when all population dynamics on the time scales $T_{i}$ and $\tau_{\mathrm{eq}}$ is complete.

Our experimental data are fitted to Eq. (A12), for which fractions $f_{i}$ are calculated in each optimization step. The lifetime $\tau_{\text {eq }}$ of the intermediate state is taken to be $0.6 \mathrm{ps}$, as was determined previously for bulk HDO: $\mathrm{D}_{2} \mathrm{O} .{ }^{44}$ We should note that the quality of the fit, the time constants $T_{i}$, and the shape of the determined pump-probe spectra $A_{i}(\omega)$ are hardly affected by the precise value of $\tau_{\text {eq }}$, because the heating signal is in general small compared to the pump-probe signal. After optimization, the fitted heating signal is subtracted from $\Delta \alpha(\omega, t)$ to obtain the pure rotation-free pump-probe signal $\Delta \alpha_{\mathrm{RF}}(\omega, t)$ :

$$
\Delta \alpha_{\mathrm{RF}}(\omega, t)=\Delta \alpha(\omega, t)-\Delta \alpha_{T}(\omega, T) .
$$

The heating signal is assumed to be isotropic, and the same functional form is subtracted from both the parallel and perpendicular absorption changes to obtain the pure pumpprobe signals $\Delta \alpha_{\|}(\omega, t)$ and $\Delta \alpha_{\perp}(\omega, t)$. These signals are used to construct the anisotropy $R(\omega, t)$ using Eq. (2).

Only data points after $0.4 \mathrm{ps}$ are considered in order not to exclude any coherent artifacts arising during time overlap between the pump and probe pulses. Since the OH-stretch absorption was pumped at its center, and the spectral width of the pump pulse was about as broad as the absorption linewidth, no significant spectral diffusion was observed. When pumping the $\mathrm{OH}$-stretch absorption at its flanks, we do observe spectral diffusion. ${ }^{46}$

No heating signal is observed for micelles of $w_{0} \leqslant 4$. For larger micelles, the heating signal is observed to increase with $w_{0}$. Its amplitude $\Delta \alpha_{T}(\omega, t)$ becomes of similar amplitude as the pump-probe signal $\Delta \alpha_{\mathrm{RF}}(\omega, t)$ at $t=5 \mathrm{ps}$ for $w_{0}$ $=8$, at $3.5 \mathrm{ps}$ for $w_{0}=15$, and at 2 ps for $w_{0}=30$. The decay 
of the thermal signal due to cooling of the micelles is well described up to $40 \mathrm{ps}$. We find $\tau_{\text {cool }}=50,80$, and $150 \mathrm{ps}$ for $w_{0}=8,15$, and 30 , respectively.

${ }^{1}$ N. E. Levinger, Science 298, 1722 (2002).

${ }^{2}$ T. K. Jain, M. Varshney, and A. Maitra, J. Phys. Chem. 93, 7409 (1989).

${ }^{3}$ J. B. Brubach, A. Mermet, A. Filabozzi, A. Gerschel, D. Lairez, M. P. Krafft, and P. Roy, J. Phys. Chem. B 105, 430 (2001).

${ }^{4}$ H. MacDonald, B. Bedwell, and E. Gulari, Langmuir 2, 704 (1986).

${ }^{5}$ D. Fioretto, M. Freda, G. Onori, and A. Santucci, J. Phys. Chem. B $\mathbf{1 0 3}$ 2631 (1999).

${ }^{6}$ M. Giustini, G. Palazzo, G. Colafemmina, M. DellaMonica, M. Giomini, and A. Ceglie, J. Phys. Chem. 100, 3190 (1996).

${ }^{7}$ Chemistry and Technology of Surfactants, edited by R. J. Farn (Blackwell, Oxford, 2006)

${ }^{8}$ S. S. Berr, J. Phys. Chem. 91, 4760 (1987).

${ }^{9}$ C. A. Bunton, F. Nome, F. H. Quina, and L. S. Romsted, Acc. Chem. Res. 24, 357 (1991).

${ }^{10}$ M. Senōo, K. Sawada, K. Araki, K. Iwamoto, and H. Kise, J. Colloid Interface Sci. 78, 57 (1980).

${ }^{11}$ C. F. Polnaszek and R. G. Bryant, J. Chem. Phys. 81, 4038 (1984).

${ }^{12}$ J. Lang, G. Mascolo, R. Zana, and P. L. Luisi, J. Phys. Chem. 94, 3069 (1989).

${ }^{13}$ A. Maitra, J. Phys. Chem. 88, 5122 (1984).

${ }^{14}$ M. Wong, J. K. Thomas, and T. Nowak, J. Am. Chem. Soc. 99, 4730 (1977)

${ }^{15}$ G. Carlström and B. Halle, Langmuir 4, 1346 (1988).

${ }^{16}$ M.-C. Belissent-Funel, S. H. Chen, and J.-M. Zanotti, Phys. Rev. E 51, 4558 (1995)

${ }^{17}$ M. R. Harpham, B. M. Ladanyi, and N. E. Levinger, J. Chem. Phys. 121, 7855 (2004).

${ }^{18}$ A. Scodinu and J. T. Fourkas, J. Phys. Chem. B 106, 10292 (2002).

${ }^{19}$ G. Seifert, T. Patzlaff, and H. Graener, Phys. Rev. Lett. 88, 147402 (2002).

${ }^{20}$ R. A. Farrer and J. T. Fourkas, Acc. Chem. Res. 36, 605 (2003).

${ }^{21}$ J. C. Deak, Y. Pang, T. D. Sechler, Z. Wang, and D. D. Dlott, Science 306, 473 (2004).
${ }^{22}$ G. M. Sando, K. Dahl, and J. C. Owrutsky, J. Phys. Chem. A 108, 11209 (2004).

${ }^{23}$ D. Cringus, J. Lindner, M. T. W. Milder, M. S. Pshenichnikov, P. Voehringer, and D. A. Wiersma, Chem. Phys. Lett. 408, 162 (2005).

${ }^{24}$ H.-S. Tan, I. R. Piletic, and M. Fayer, J. Chem. Phys. 122, 174501 (2005).

${ }^{25}$ A. M. Dokter, S. Woutersen, and H. J. Bakker, Phys. Rev. Lett. 94, 178301 (2005).

${ }^{26}$ I. R. Piletic, D. E. Moilanen, D. B. Spry, N. E. Levinger, and M. Fayer, J. Phys. Chem. A 110, 4985 (2006).

${ }^{27}$ A. M. Dokter, S. Woutersen, and H. J. Bakker, Proc. Natl. Acad. Sci. U.S.A. 103, 15355 (2006).

${ }^{28}$ J. Faeder and B. M. Ladanyi, J. Phys. Chem. B 194, 1033 (2000).

${ }^{29}$ P. K. Das, G. V. Srilakshmi, and A. Chaudhuri, Langmuir 15, 981 (1999).

${ }^{30}$ I. M. Cuccovia, L. G. Dias, F. A. Maximiano, and H. Chaimovich, Langmuir 17, 1060 (2001).

${ }^{31}$ P. A. Bergstrom, J. Lindgren, and O. Kristiansson, J. Phys. Chem. 95, 8575 (1991)

${ }^{32}$ M. F. Kropman and H. J. Bakker, Science 291, 2118 (2001).

${ }^{33}$ M. F. Kropman and H. J. Bakker, J. Am. Chem. Soc. 126, 9135 (2004).

${ }^{34}$ S. Berr, R. R. M. Jones, and J. S. Johnson, J. Phys. Chem. 96, 5611 (1992).

${ }^{35}$ C. F. Wu, S. H. Chen, L. B. Shih, and J. S. Lin, Phys. Rev. Lett. 61, 645 (1988).

${ }^{36}$ D. Stigter, J. Phys. Chem. 68, 3603 (1964).

${ }^{37}$ M. R. Harpham, B. M. Ladanyi, and N. E. Levinger, J. Phys. Chem. B 109, 16891 (2005)

${ }^{38}$ J. Faeder, M. V. Albert, and B. M. Ladanyi, Langmuir 19, 2514 (2003).

${ }^{39}$ S. Raugei and M. L. Klein, J. Chem. Phys. 116, 196 (2001).

${ }^{40}$ B. Derecskei, A. Derecskei-Kovacs, and Z. Schelly, Langmuir 15, 1981 (1999).

${ }^{41}$ C. J. Fecko, J. D. Eaves, J. J. Loparo, A. Tokmakoff, and P. L. Geissler, Science 301, 1698 (2003).

${ }^{42}$ C. J. Fecko, J. J. Loparo, S. T. Roberts, and A. Tokmakoff, J. Chem. Phys. 122, 054506 (2005).

${ }^{43}$ G. Lipari and A. Szabo, Biophys. J. 30, 489 (1980).

${ }^{44}$ Y. L. A. Rezus and H. J. Bakker, J. Chem. Phys. 125, 144512 (2006).

${ }^{45}$ M. F. Kropman, H.-K. Nienhuys, and H. J. Bakker, Phys. Rev. Lett. 88, 077601 (2002).

${ }^{46}$ A. M. Dokter, S. Woutersen, and H. J. Bakker (unpublished). 